Akute Atemwegsinfektionen

\title{
Konservativer Start der Antibiotikatherapie reicht meist aus
}

Patienten mit akuten Atemwegsinfektionen, etwa einer exazerbierten COPD oder Pneumonie, werden immer häufiger bereits initial mit Breitspektrum-Antibiotika behandelt. Das ist unnötig und fördert lediglich Resistenzen, hieß es beim DGP-Kongress in Leipzig.

Bei Patienten mit akut exazerbierter COPD plädierte Prof. Rainer Hauck, Chefarzt in den Kreiskliniken Altötting-Burghausen, für einen konservativen Start der Antibiotikatherapie bzw. eine Therapie orientiert am Risikoprofil. Laut einer retrospektiven Zehnjahres-Untersuchung hat sich bei AECOPD-Patienten mit Infektion der unteren Atemwege (Bronchitis oder Pneumonie) der Einsatz von Breitband-Antibiotika wie Aminoglykosiden oder Ciprofloxacin verdreifacht, berichtete er (Planquette B et al., Int J COPD 2015;10:379-88). Dies habe häufig irrationale Gründe. So war in der Studie eine Beatmung ein Prädiktor für ein Breitspektrum-Antibiotikum, nur bei $14 \%$ habe sich die Wahl des Antibiotikums nach Risikofaktoren für eine Besiedelung mit Pseudomonas aeruginosa gerichtet.

$\mathrm{Zu}$ den Risikofaktoren für eine solche Infektion zählt eine schwere COPD mit einem $\mathrm{FEV}_{1}$-Wert $<35 \%$, eine systemische Steroidtherapie länger als vier Wochen in einer Dosis $>10 \mathrm{mg}$, eine Antibiose innerhalb der letzten drei Monate sowie ein zurückliegender Krankenhausaufenthalt, erinnerte Hauck.

\section{Prognose mit initialem Breitspektrum-Antibiotikum nicht verbessert}

Ein konservativer Start der Antibiotikatherapie bedeute keine Verschlechterung der Prognose von Patienten mit AECOPD, sagte Hauck. Bei den Studienteilnehmern war die Prognose von Patienten, die initial mit einem Antibiotikum mit Pseudomonas-Wirksamkeit behandelt wurden, sogar schlechter als die von Patienten, die mit einem dafür ungeeigneten Antibiotikum behandelt wurden (Therapieversagen 35 vs. $27 \%$ ).

Auch bei Patienten mit leichter bis mittelgradiger ambulant erworbener Pneumonie (CAP) sei eine Beta-Laktam-Monotherapie einer Kombination von Beta-Laktam-Antibiotikum / Makrolid bzw. Therapie mit einem Fluorchinolon nicht unterlegen, betonte Prof. Stefan Krüger, Chefarzt am Florence-NightingaleKrankenhaus in Düsseldorf. In einer Studie bei 2.283 CAP-Patienten im Alter von 70 Tagen war die 90-Tage-Mortalität mit $9 \%$ bis $11 \%$ in allen drei Gruppen ähnlich (Postma D et al., N Eng J Med 2015; 372:1312-23).

Glukokortikoide erhöhen Resistenz von H. influenzae Nicht selten besteht bei COPD-Patienten mit schweren AECOPD allerdings auch eine bakterielle Multiresistenz. Dazu können offenbar sogar die Medikamente beitragen, die Exazerbationen vorbeugen sollen wie Glukokortikoide. Glukokortiko- ide modulieren den Biofilm von Haemophilus influenzae (H.i.), einem häufigen Erreger von Atemwegsinfektionen, und können auf diese Weise die Antibiotikatoleranz erhöhen, erklärte Hauck. Vermittelt wird dieser Effekt durch eine Beeinflussung von H.i.-Genen durch Glukokortikoide. Durch Veränderung der H.i.-Genexpression wird die Biofilm-Bildung beeinflusst, die bakterielle Persistenz erhöht und die Antibiotikatoleranz beeinflusst. Dieser Effekt wurde nach Angaben von Hauck bei Invitro-Untersuchungen mit infizierten Lungenzellen sowohl von Asthma- als auch von COPD-Patienten bei Zugabe von Beclomethason und Prednisolon nachgewiesen. Weniger Einfluss hatte Mometason.

Roland Fath

Quelle: 57. Kongress der Deutschen Gesellschaft für Pneumologie, Leipzig, 3. März 2016

\section{Proteasom hemmen, Heilung fördern?}

Das Immunoproteasom ist ein Proteinkomplex, der vor allem in Immunzellen vorliegt und dazu beiträgt, Proteine in kleinere Bestandteile zu zerlegen.

Forscher haben nun herausgefunden, dass das Immunoproteasom die Makrophagen der Lunge in ihrer Reparaturfunktion hemmt, heißt es in einer Mitteilung des Helmholtz Zentrums München. Dies biete neue Therapieoptionen: Eine spezifische Hemmung des Immunoproteasoms könnte Heilungsprozesse der Lunge begünstigen.

Das Team fand heraus, dass das Immunoproteasom die IL-4 Stimulation der Makrophagen reguliert (Cell Death Differ 2016; online 18. März). Dabei hemmt es den IL-4-Signalweg und bremst damit deren alternative Aktivierung. „Bei Experimenten mit Lungenmakrophagen konnten wir zeigen, dass in Zellen, die kein Immunoproteasom besitzen, ein bestimmter Rezeptor für IL-4 angereichert wird", wird Ilona Kammerl zitiert, eine der Erstautoren der Publikation.

Das Immunoproteasom wirke somit hemmend auf den IL-4 Signalweg und limitiert so dessen Wirkung. Ein pharmakologischer Immunoproteasom-Inhibitor sollte die Hemmung des IL-4 Signalweges aufheben und so den Makrophagen erlauben, verstärkt in den Reparatur- und AufräumModus zu schalten.

"In der Tat konnten wir durch Zugabe des Inhibitors eine signifikant stärkere alternative Aktivierung der Lungenmakrophagen beobachten", wird Studienleiter Tobias Stöger in der Mitteilung zitiert.

Die Wissenschaftler wollen jetzt im Tiermodell herausfinden, ob eine gezielte Behandlung mit spezifischen Inhibitoren des Immunoproteasoms die Heilungsvorgänge in der Lunge beschleunigt, etwa nach einer akuten Lungenentzündung. Entsprechende Vorexperimente laufen bereits. (eb) 